\title{
ÉTUDE PRÉLIMINAIRE DES CARACTÉRISTIQUES DE TRAITE DES VACHES LAITIÈRES
}

\author{
A. DESVIGNES et M. POUTOUS \\ Station centrale de Génétique animale, \\ Centre national de Recherches zootechniques, Jony-en-Josas (Seine-et-Oise)
}

\section{SOMMAIRE}

Des contrôles de traite ont été effectués de 1956 à I959 sur un troupeau de vaches laitières de race Brune des Alpes. I 207 données provenant de 77 animaux différents ont été analysées.

Les différents critères employés (quantité totale de lait, durée totale de traite, débit maximum par minute, date du débit maximum depuis le début de la traite, quantités de lait obtenues au cours des 3 ou 4 premières minutes de traite en pourcentage de la quantité totale) présentent des répétabilités relativement faibles; on a étudié les principaux facteurs susceptibles de faire varier ces caractéristiques.

$\left.\mathrm{r}^{0}\right)$ La quantité totale de lait recueillie à la traite est en corrélation étroite avec les autres critères ; cependant, une correction tenant compte de la quantité de lait n'améliore pas sensiblement la répétabilité.

$\left.2^{\circ}\right)$ L'époque de nos contrôles a eu une influence importante sur la variation de nos données; cela tient aux changements des conditions de traite survenus au cours des années. Le caractère qui a été le moins affecté est le pourcentage de lait obtenu au cours des 3 premières minutes de traite.

$\left.3^{\circ}\right)$ I.e numéro de lactation est une source de variation des critères que nous avons étudiés, mais il semble intervenir de façon indirecte, par son action sur la quantité totale de lait recueillie à la traite.

$\left.4^{\circ}\right)$ Le stade de lactation a une influence non négligeable sur les caractéristiques de traite; un contrôle réalisé au cours de la première moitié de la lactation permet une meilleure connaissance de l'aptitude à la traite d'un animal.

Les relations des critères entre eux, et des critères de traite avec les critères de lactation, montrent enfin qu'il est possible d'envisager une sélection sur l'aptitude à la traite, sans aller à l'encontre d'une amélioration génétique de la production laitière.

\section{INTRODUCTION}

Actuellement, la gestion d'un troupeau de vaches laitières se pose en termes économiques : il s'agit pour l'éleveur, dans une conjoncture de commercialisation donnée, d'obtenir un revenu maximum de son cheptel exploité ; cela implique une 
augmentation du rapport global de l'étable, fonction du nombre et du niveau de performances unitaires, mais cela exige aussi une diminution des frais de production.

Parmi ces derniers, le salaire du ou des ouvriers responsables de la conduite du troupeau constitue un poste important. Il s'agit donc d'assurer à la main-d'œuvre utilisée une efficience maximum ; la généralisation de la traite mécanique, couplée la plupart du temps à des installations de stabulation libre et de salle de traite de divers types, vise à ce que le nombre d'animaux traits à l'heure, d'une façon correcte, dans des conditions d'hygiène satisfaisantes, soit le plus élevé possible. Les journées de 1'Association Française de Zootechnie étaient d'ailleurs consacrées en Ig6o à cet important problème (A. F. Z., I960).

On cherche donc à avoir un troupeau de vaches ayant un tempérament de traite facile, et en quelque sorte standard; on réduit ainsi le temps de travail du vacher et on normalise son intervention sur un cheptel homogène.

Il est apparu, par ailleurs, clairement que les différences dans le comportement à la traite des vaches étaient partiellement d'origine génétique. Lue récent Congrès international de Zootechnie de Hambourg en donne de nombreux exemples. Dans les programmes d'amélioration génétique appliqués aux bovins laitiers, on a donc tenu compte de plus en plus du caractère " aptitude à la traite " en lui-même et non plus indirectement comme par le passé, en invoquant le rôle d'une "bonne gymnastique fonctionnelle " de la mamelle, sur le niveau des performances réalisées.

Nous nous contenterons, dans ce mémoire préliminaire d'apporter les premiers résultats que nous avons acquis en matière de contrôle de l'aptitude à la traite des vaches laitières d'un troupeau dont nous assurons la gestion technique; à partir d'eux, nous essaierons de dégager des méthodes de travail pour le futur, plus rationnelles, applicables à vaste échelle, notamment dans le cadre du programme de testage des taureaux d'insémination artificielle.

\section{MATÉRIEL ET MÉTHODES}

Les contrôles ont été effectués de 1956 à 1959 sur le troupeau de la ferme de l'Abbave de la Pierre-qui-Vire dans l'Yonne. Ce troupeau se compose en moyenne de $3 \circ$ vaches adultes, toutes de race Brune des Alpes, qui sont entretenues dans les conditions normales d'une bonne exploitation commerciale de la région. Maintenues en stabulation libre l'hiver et au pâturage pendant le reste de l'années, les vaches sont constamment traites dans une salle à 3 postes équipés en matériel Gascoigne. L'égouttage se fait entièrement à la machine. Toutes les vaches en lactation, en bon état sanitaire ont été contrôlées avec une périodicité moyenne de i mois. On notait, pour chaque animal, la quantité de lait obtenue depuis le début de la traite, jusqu'à la fin de la ${ }^{\mathrm{re}}, 2^{\mathrm{e}} \ldots n^{\text {iémo }}$ minute, et la durée totale de la traite.

On estimait que la traite débutait au moment de la pose du $3^{\mathrm{e}}$ gobelet et qu'elle se terminait lors de l'enlèvement de ce même gobelet ; elle englobait ainsi les opérations d'égouttage à la machine. Les quantités étaient mesurées volumétriquement, par référence à une réglette graduée, visible a travers les parois transparentes du pot trayeur. Dans nos conditions d'observation, une source d'erreur provient de la mousse abondante qui se produit en cours de traite et qui rend parfois diffcile l'appréciation du niveau du lait.

Au total, I 207 données ont été retenues, provenant de 77 animaux différents. Elles ont été mises en cartes perforées et traitées par un programme spécial sur ordinateur I. B. M. 650. P'our chaque carte, les critères suivants ont été calculés :

- Durée totale de traite (en $\mathrm{mn}$ ).

- Débit maximum par minute (en $\mathrm{kg} / \mathrm{mn}$ ). Les débits par minute ont été obtenus par simple différence entre les quantités de lait au début et à la fin de la minute considérée. 
- Temps du débit maximum (en $\mathrm{mn}$ ), égal par convention au numéro d'ordre de la minute pendant laquelle le débit était maximum diminué de $0,5^{\circ}$.

- Pourcentage de lait obtenu au bout des 3 premières minutes, par rapport à la quantité totale de lait de la traite (ou pourcentage en 3 minutes).

- Pourcentage de lait obtenu en 4 minutes (pourcentage en 4 minutes) défini de manière analogue au critère précédent.

- Quantité totale de lait de la traite.

La durée totale a été prise comme critère général d'aptitude à la traite.

les courbes de traite exprimées en débits cumulés ou en débits minute par minute. (graph. I), donnent une représentation de ces différents critères.
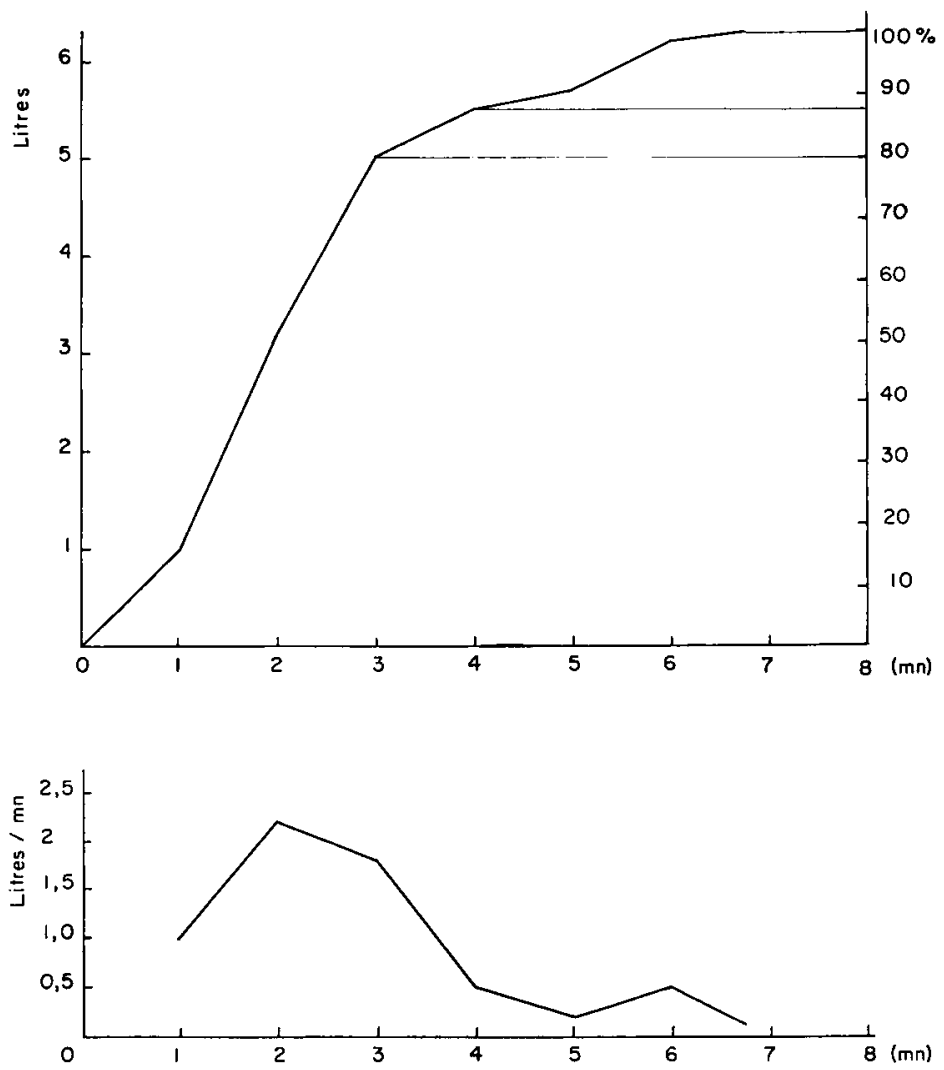

Graphique I. - Exemple de courbe de traite - en débits cumulés - en débils minute par minute Les critères retenus sont : la quantité de lait de la traite, la durée totale de la traite, le débit maximum par minute, le temps du débit maximum, le pourcentage de lait obtenu au bout des 3 premières minutes, le pourcentage obtenu au bout des 4 premières minutes.

\section{RÉSULTATS ET DISCUSSION}

\section{I. - VALEUR MOYENNF, ET RÉPARTITION DES CRITÈRES}

Les valeurs moyennes, les écarts-types et les coefficients de variation des critères ont été rassemblés dans le tableau I, et les courbes de répartition des données sur les graphiques $2 \mathrm{~A}$ à $2 \mathrm{~F}$. 
TABLEAU I

Caractéristiques moyennes des critères de traite

\begin{tabular}{|c|c|c|c|c|}
\hline & Lnité & Moyenne & Ecart-type & $\begin{array}{l}\text { Coefficient } \\
\text { de variation }\end{array}$ \\
\hline Quantité de lait de la traite .... & litres & 6,07 & 2,32 & $38 \%$ \\
\hline Temps total de traite........... & minutes & 6,53 & 2,31 & $36 \%$ \\
\hline Temps clébit maximum. & minutes & 1,48 & 1,03 & $70 \%$ \\
\hline 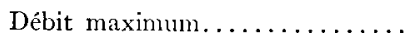 & $1 / \mathrm{mn}$ & 2,01 & 0,70 & $35 \%$ \\
\hline$\% 3^{\mathrm{e}}$ minute $\ldots \ldots \ldots \ldots \ldots$ & $\%$ & 71,92 & $20,1: 2$ & $28 \%$ \\
\hline$\%$ to $^{\mathrm{e}}$ minute. & $\%$ & $8: 3,50$ & 16,30 & $20 \%$ \\
\hline
\end{tabular}

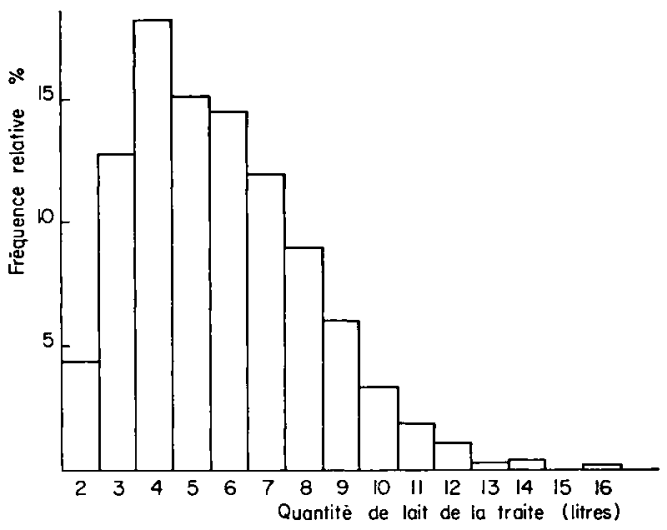

Graphique $2 a$

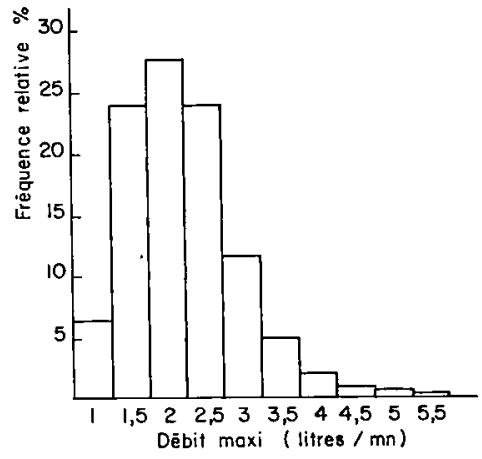

GrapiIIQUe $2 c$

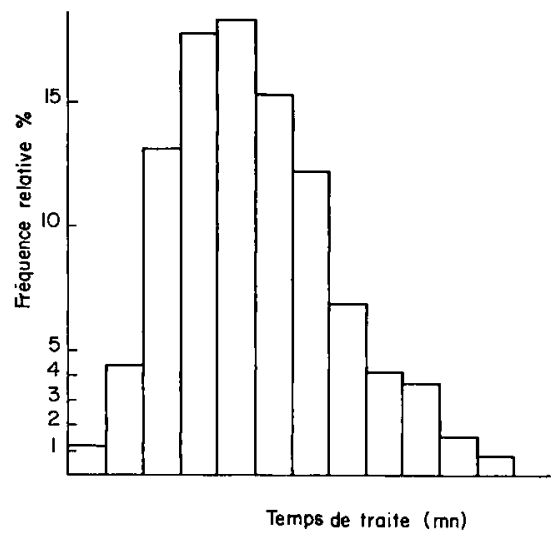

GRAPHOQUE $2 b$

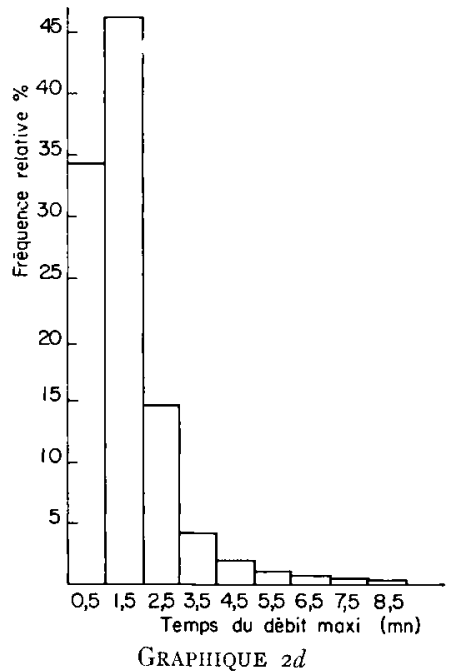


Le coefficient de variation très élevé du temps du débit maximum s'explique par la relative imprécision de ces mesures obtenues à la ferme. Le critère le moins variable est le pourcentage en 4 minutes : à ce moment, un pourcentage important de vaches ont terminé leur traite. Le coefficient de variation des autres paramètres est du même ordre de grandeur : il est relativement élevé, ce qui indique une grande variabilité des caractères étudiés. Les répartitions sont toutes fortement dissymétriques; nous avons montré, par ailleurs, qu'elles diffèrent nettement de répartitions laplaciennes, et obtenu des transformations permettant de les normaliser.

La comparaison de nos résultats avec ceux des auteurs ayant étudié le même problème est souvent délicate. Non seulement les méthodes de recueil des données et les conditions d'exploitation des animaux diffèrent largement, mais aussi les

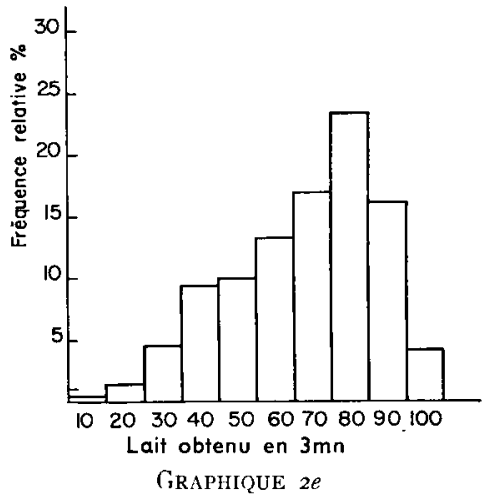

GRAPHIQUES 2. - Diagrammes de répartitions des 1207 données classées suivant les divers critères de traite

définitions des critères ne sont souvent pas les mêmes. Par exemple, le débit maximum peut être calculé à partir de courtes périodes de contrôle (30 ou $\mathrm{I}_{5} \mathrm{~s}$ ); il présente alors des valeurs plus élevées que dans le cas présent. I 'âge et le stade de lactation des animaux contrôlés varient aussi, beaucoup de chercheurs ayant utilisé des primipares dans les premiers mois de leur lactation. Quoi qu'il en soit, il semble exister, pour le débit maximum et pour le pourcentage en 3 minutes, une concordance satisfaisante avec les constatations des travaux similaires (JoHAxsson, I96I; Politiek, I96I ; SANDvik, I957). Par contre, la durée de traite moyenne que nous avons observée paraît supérieure à la plupart des résultats des auteurs étrangers, sauf cas particulier (DODD, I953). La différence s'explique, pensons-nous, par l'habitude des vachers de la ferme d'égoutter très longuement les animaux.

\section{2. - RÉPÉTABILITÉ DES CRITÈrRES}

Les coefficients de répétabilité des critères (tabl. 2) ont été obtenus par la méthode classique de décomposition de la variance à une voie, les mesures étant classées d'après l'animal sur lequel elles avaient été obtenues. 
TABIEAU 2

Coefficients de répétabilité des critères de traite

\begin{tabular}{|c|c|}
\hline Ouantité totale de lait de la traite ....... & 0,21 \\
\hline Durée totale...................... & 0,31 \\
\hline Temps de débit maximum ............ & 0,29 \\
\hline 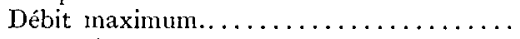 & 0,38 \\
\hline$\% 3$ minutes $\ldots \ldots \ldots \ldots \ldots \ldots \ldots \ldots$ & $0,5,2$ \\
\hline$\% 4$ minutes $\ldots \ldots \ldots \ldots \ldots \ldots \ldots \ldots$ & 0,17 \\
\hline
\end{tabular}

Ces coefficients de répétabilité peuvent être considérés comme un coefficient de corrélation moyen entre 2 mesures quelconques d'un même animal. De ce fait, ils indiquent l'importance relative des facteurs liés à l'animal par rapport à l'ensemble des causes de variation. Les critères les plus intéressants sont les deux pourcentages, puis le débit maximum et le temps total.

Ces valeurs sont sensiblement inférieures à celles qui sont citées par ailleurs. Par exemple, BEck et al. (I95I) ont relevé des répétabilités supérieures à 0,80 pour des mesures effectuées à un jour d'intervalle, et même d'une lactation à la suivante. Ces différences s'expliquent en partie par les méthodes de contrôle et de calcul des critères que nous avons employées, en particulier pour le débit maximum. Nous pensons néanmoins qu'on doit surtout tenir compte des nombreuses variations dans la technique de traite qui se produisent normalement en plusieurs années dans une ferme de ce type. D'ailleurs les valeurs de répétabilité trouvées dans des enquêtes effectuées dans des élevages ordinaires sont plus faibles qu'en station (SANDVIK, I957 a).

\section{3. - REI ATIONS AVEC LA QUANTITÝ DE LAIT PAR TRAITE}

Après répartition des données en 4 groupes d'après la quantité totale de lait à la traite, nous avons calculé pour chaque catégorie les valeurs moyennes des débits minute par minute. Les courbes obtenues (graph. 3) diffèrent nettement entre elles ainsi que les paramètres qui les définissent.

Il existe effectivement des variations systématiques des lignes de régression de ces paramètres en fonction du niveau de production (graph. 4 et 5 ). Le sens et l'intensité de ces relations de dépendance peuvent être estimés par les coefficients de corrélation et de régression linéaires rassemblés dans le tableau 3.

Les critères les plus liés à la quantité totale de lait sont le débit maximum $(r=0,56)$ et la durée totale $(r=0,40)$. Cette dernière augmente de 0,40 minute par litre de lait supplémentaire. Les pourcentages eux-mêmes ne sont pas entièrement indépendants du niveau de production. Pour vérifier si les relations étudiées s'expliquent effectivement par les régressions linéaires, nous avons d'abord calculé les valeurs moyennes des critères par classes de I litre de la quantité totale de lait. 


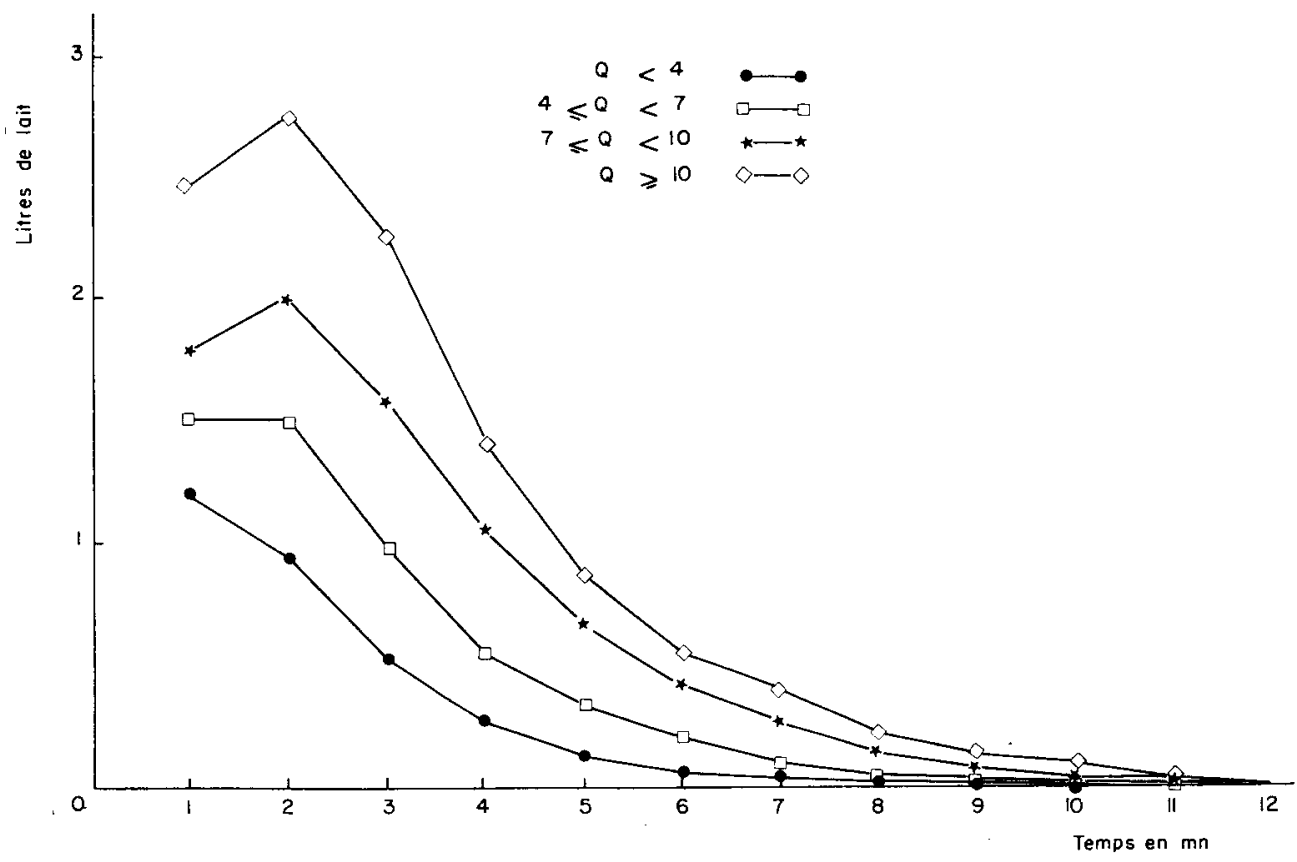

GRAPHIQUe 3. - Courbes moyennes de traite a divers niveaux de production totale $Q$.

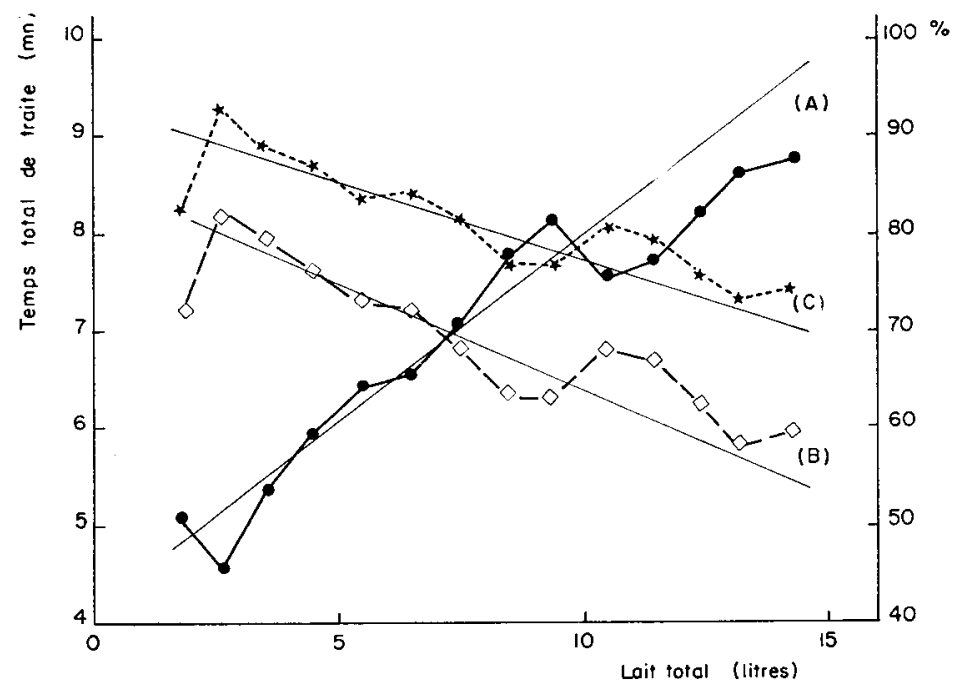

GRAPHIQUE 4. - Valeurs moyennes et droite de régression

A - du temps total de traite,

$\mathrm{B}-d u$ pourcentage de lait obtenu en 3 minutes,

$\mathrm{C}-d u$ pourcentage de lait obtenu en 4 minutes par classes de 1 litre de lait total a la traite. 
L'analyse de la variance de ces moyennes permet d'estimer la réduction de la somme des carrés due à l'ajustement sur la vraie ligne de régression des variables dépendantes sur la variable indépendante. Pour nos données, la réduction à un ajustement linéaire était significativement plus faible pour le temps total et le débit

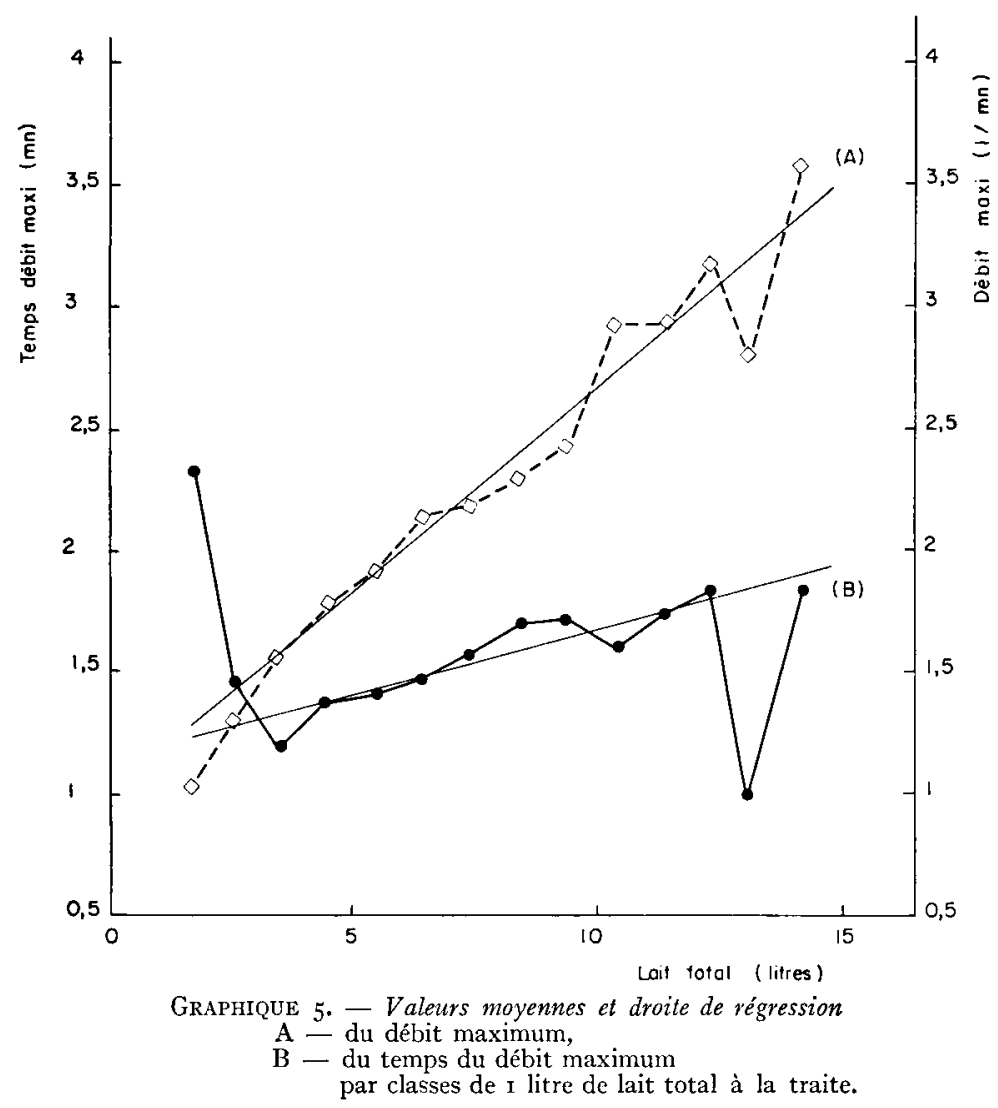

TABLEAU 3

Coefficients de corrélation ( $\mathrm{r}$ ) et de régression (b) linćaires des critères sur la quantité totale de lait à la traite (en litres)

\begin{tabular}{|c|c|c|c|c|c|}
\hline & $\begin{array}{l}\text { Temps total } \\
(\mathrm{mn})\end{array}$ & $\begin{array}{l}\text { Temps du } \\
\text { débit maxi- } \\
\text { mum (mn) }\end{array}$ & $\begin{array}{l}\text { Débit maxi- } \\
\text { mum }(1 / \mathrm{mm})\end{array}$ & $\% \underset{(\%)}{3 \text { minutes }}$ & $\begin{array}{c}\% \text { m minutes } \\
(\%)\end{array}$ \\
\hline $\begin{array}{l}\text { Coefficient de corrélation } \\
\quad(r) \ldots \ldots \ldots \ldots \ldots \ldots \ldots\end{array}$ & 0,40 & 0,13 & 0,56 & $-0,26$ & $-0,24$ \\
\hline $\begin{array}{l}\text { Coefficient de régression } \\
\qquad(b) \ldots \ldots \ldots \ldots \ldots \ldots \ldots\end{array}$ & $0,{ }^{\prime} 10$ & 0,06 & 0,17 & $-2,2$ & $-1,7$ \\
\hline
\end{tabular}


maximum, de telle sorte que pour ces deux critères une équation linéaire ne semble pas suffisante. Néanmoins l'augmentation du coefficient de corrélation que procurerait un ajustement sur la ligne des moyennes est pratiquement négligeable pour le débit maximum $(+0,01)$, et reste faible pour le temps total $(+0,03)$. L'introduction d'un terme du $2^{e}$ degré dans les équations de prédiction ne nous a donc pas paru justifiée, compte tenu des calculs importants qu'elle aurait entraînés. On peut vérifier sur les graphiques 4 et 5 la précision de l'ajustement linéaire.

Après correction des données pour les ramener à une quantité constante ( 6 litres) de lait à la traite, nous avons calculé les répétabilités des caractéristiques (tabl. 4).

TABLEAU 4

Répétabilités des caractéristiques de traite corrigées pour la quantité de lait

\begin{tabular}{|c|c|}
\hline Temps total ... & 0,37 \\
\hline Temps du débit maximum............. & 0,23 \\
\hline 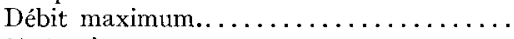 & 0,41 \\
\hline$\% 3$ minutes $\ldots \ldots \ldots \ldots \ldots \ldots \ldots \ldots$ & 0,55 \\
\hline$\% 4$ minutes...$\ldots \ldots \ldots \ldots \ldots \ldots \ldots$ & 0,48 \\
\hline
\end{tabular}

L'efficacité de la correction ne doit pas être jugée uniquement d'après la faible augmentation ( 3 p. roo) des répétabilités qu'elle procure. En effet, elle élimine aussi bien les différences entre animaux dues à des productions moyennes variables que les différences intra-animaux. Pour des contrôles effectués tous les mois, l'augmentation est faible. On doit cependant remarquer que le but de la correction est de comparer des vaches contrôlées un nombre de fois aussi restreint que possible et à des niveaux de production qui seront en pratique difficiles à standardiser. Des comparaisons valables ne pourront être faites que si cette cause de variation est éliminée.

\section{4. - Infl,uence, de, ia date de Controle,}

Nous avons déjà dit que de 1956 à 1959 plusieurs changements importants se sont produits dans la technique de traite et les conditions du milieu. L'observation directe montre bien par ailleurs que d'une traite à l'autre, il existe des variations souvent sensibles dans le comportement tant des trayeurs que des animaux. Dans 1a mesure où ces facteurs ont le même effet sur tous les animaux contrôlés à la même date, une correction basée sur la moyenne d'étable permettrait de les éliminer. Pour tester cette hypothèse, nous avons effectué une analyse de variance à I voie d'après les dates des relevés. Tous les effets dates étant significatifs, nous avons ensuite calculé les pourcentages de la variance totale due aux dates, à la fois sur les données brutes et les données corrigées pour la production de lait (tab1. 5).

Le temps total de traite est le plus sensible aux variations systématiques entre dates, même après correction pour la quantité de lait. Par contre, les pourcentages et le débit maximum sont relativement indépendants. Ces analyses supposent que 
TABLEAU 5

Pourcentage de la variance totale due à des différences entre dates

\begin{tabular}{|c|c|c|}
\hline & $\begin{array}{c}\text { variables } \\
\text { brutes }\end{array}$ & $\begin{array}{l}\text { variables } \\
\text { corrigées pour la } \\
\text { quantité de lait }\end{array}$ \\
\hline 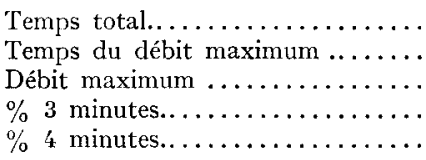 & $\begin{aligned} & 23 \% \\
& 10 \% \\
& 14 \% \\
& 8 \% \\
& 6 \%\end{aligned}$ & $\begin{aligned} & 13 \% \\
& 10 \% \\
& 5 \% \\
& 9 \% \\
& 8 \%\end{aligned}$ \\
\hline
\end{tabular}

l'aptitude à la traite des animaux soit restée la même en moyenne pendant toute cette période. Bien qu'aucune sélection directe n'ait été faite, il est cependant possible qu'une sélection indirecte ait eu lieu par élimination des faibles productrices ou des vaches atteintes de mammites. Par ailleurs, le modèle que nous avons utilisé suppose que le $j^{\text {iime }}$ contrôle effectué à la date $i$ a pour valeur

$$
y_{i j}=m+d_{i}+e_{i j},
$$

$m$ étant la moyenne générale, $d_{i}$ l'influence réelle de la date, $e_{i j}$ une valeur résiduelle aléatoire indépendante. I'hypothèse d'indépendance ne serait vérifiée que s'il n'existait pas des réactions différentes entre animaux, c'est-à-dire, en termes statis-, tiques, que si l'interaction dates-vaches était nulle. De plus, les vaches n'ayant pas toutes été contrôlées à toutes les dates, il existe une relation entre contrôles consécutifs due au fait que l'on y retrouve en moyenne les mêmes animaux. Pour corriger cette erreur, nous avons calculé les répétabilités des caractéristiques de traite corrigées pour la quantité de lait et ramenées à une durée moyenne par date constante (tabl. 6).

\section{TABLEAU 6}

Répétabilités des données corrigées pour la quantité totale de lait et ramenées à une durée moyenne des contrôles par date constante

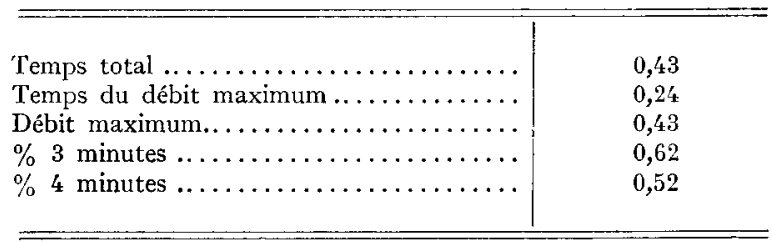

Les répétabilités calculées après élimination théorique des différences entre dates sont supérieures à ces valeurs observées de I à 4 p. Ioo seulement. L'erreur introduite par la non-orthogonalité du schéma que nous avons utilisé ne paraît 
donc pas être très importante. Compte tenu de l'augmentation notable de la fidélité des mesures qu'elle provoque, surtout pour le temps total, la correction par date ne serait pas à négliger dans le cas de la ferme de la Pierre-qui-Vire.

\section{5. - INFLUENCE dU NUMÉRo DE IACTATION}

Cette influence a été estimée par l'augmentation du coefficient de répétabilité due à 1'élimination des différences entre lactations. La décomposition de la variance a été faite d'après la méthode $n^{\circ}$ II de HENDERSON (I953), en considérant l'effet numéro d'ordre de la lactation comme fixé et la valeur réelle des animaux comme aléatoire. Les coefficients de répétabilité obtenus sont reportés dans le tableau 7 , et les différences entre lactations, la $4^{\mathrm{e}}$ étant prise comme référence, dans le tableau 8.

\section{TABLEAU 7}

Coefficients de répétabilité des caractères de traite après élimination des effets dus aux numéros de lactation (valeurs brutes)

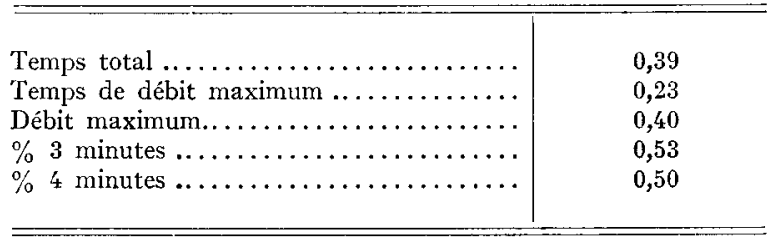

TABLEAU 8

Estimations des effets dus aux numéros de lactation (la quatrième étant prise comme référence) sur les caractéristiques de traite

\begin{tabular}{|c|c|c|c|c|c|c|}
\hline & Lait total & Temps total & $\begin{array}{l}\text { Temps du } \\
\text { débit maxi- } \\
\text { mum }\end{array}$ & $\begin{array}{c}\text { Débit } \\
\text { maximum }\end{array}$ & $\% 3$ minutes & $\% 4$ minutes \\
\hline 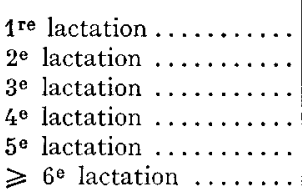 & $\begin{array}{r}-3,1 \\
-1,8 \\
-0,4 \\
0 \\
-0,6 \\
-1,6\end{array}$ & $\begin{array}{r}-1,9 \\
-0,7 \\
-0,4 \\
0 \\
0,6 \\
1,2\end{array}$ & $\begin{array}{r}-0,2 \\
-0,2 \\
-0,1 \\
0 \\
0,3 \\
-0,2\end{array}$ & $\begin{array}{r}-0,3 \\
-0,1 \\
0 \\
0 \\
0,2 \\
0,4\end{array}$ & $\begin{array}{r}13,4 \\
4,4 \\
3,6 \\
0 \\
-5,6 \\
-1,4\end{array}$ & $\begin{array}{r}11,6 \\
5,0 \\
4,7 \\
0 \\
-3,3 \\
-1,5\end{array}$ \\
\hline $\begin{array}{l}\text { Différence maxima en } \\
\text { de l'écart-type ...... }\end{array}$ & tage & 1,3 & 0,3 & 0,7 & 1,0 & 0,9 \\
\hline
\end{tabular}

Les diffërences entre $n^{0}$ de lactation, bien que statistiquement significatives, ne sont réellement importantes que pour le temps total et le pourcentage de lait en 3 minutes. L'augmentation des coefficients de répétabilité se produit surtout pour le temps total ( 7 p. IOO). 
Ces valeurs sont semblables à celles calculées sur les données corrigées pour la quantité de lait par traite, ce qui tendrait à montrer que l'effet de lactation n'est en réalité dû qu'à des différences de production. Pour vérifier de façon simple cette hypothèse, nous avons calculé (tabl. 9) les valeurs des critères, estimées après élimination des différences entre les productions de lait.

\section{TABLEAU 9}

Valeurs estimées des effets $n^{\circ}$ de lactation, après correction des variations des quantités de lait par traite

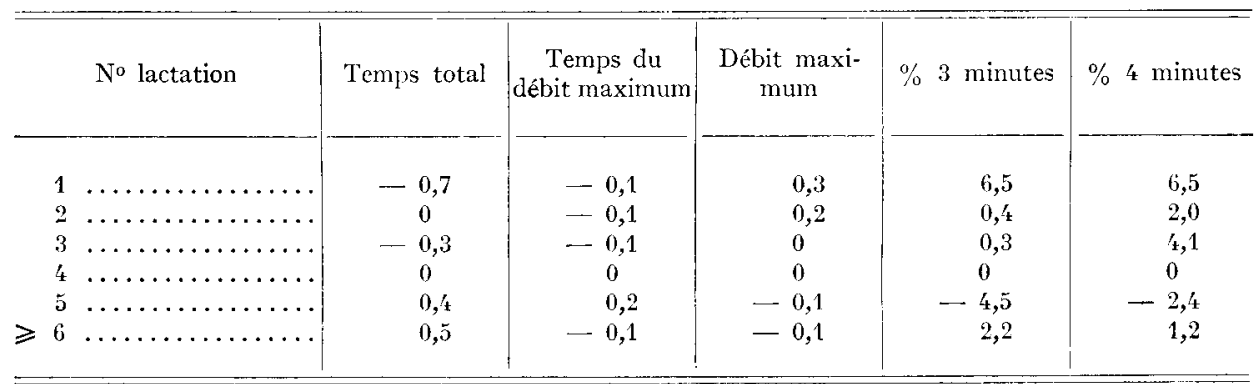

Les animaux en I $^{\text {re }}$ lactation sont plus rapides à traire, avec un débit maximum plus élevé et un pourcentage de lait en 3 et 4 minutes plus grand. Sauf pour le débit maximum, les vaches en $2^{\mathrm{e}}$ lactation ne se différencient pratiquement pas des adultes. Sans correction il est préférable de limiter les comparaisons à des animaux en $I^{\text {re }}$ lactation ou aux vaches en $2^{\mathrm{e}}$ lactation et au-delà.

\section{6. - INFLUENCE DU STADE DE LACTATION}

L'organisation d'un système de mesure de l'aptitude à la traite sera plus commode s'il suffit de quelques visites annuelles par élevage, les frais étant sensiblement proportionnels au nombre de passages. Cette simplification suppose que les animaux puissent être contrôlés à des stades de lactation différents, sauf peut-être dans quelques zones où les vêlages sont concentrés sur une courte période. Pour vérifier le bien-fondé de cette hypothèse nous avons utilisé les résultats de 27 génisses contrôlées tous les mois pendant les 9 premiers mois de leur I ere lactation. Les valeurs moyennes des critères aux $2^{\mathrm{e}}, 5^{\mathrm{e}}$ et $8^{\mathrm{e}}$ mois et pour la lactation totale ont été calculées (tab1. Io).

Sur l'échantillon des données étudié il n'existe pas de différences systématiques importantes entre les différents stades de lactation, du moins pour les valeurs ramenées à production totale par traite constante. Ces résultats sont en accord avec ceux de Johansson (I96I) et SANDvik (I957 a) alors que DoDd (I953) trouvait une diminution des débits dans la $2^{\mathrm{e}}$ partie de la lactation.

Même si elles n'influencent pas de façon systématique les valeurs moyennes des contrôles, certaines périodes pourraient être préférables, parce que permettant 


\section{TABLEAU IO}

Valeurs moyennes des caractères de traite des génisses pour le $2^{\mathbf{e}}, 5^{\mathbf{e}}$ et $8^{\mathrm{e}}$ mois et la lactation totale

\begin{tabular}{|c|c|c|c|c|c|c|}
\hline & $\begin{array}{l}\text { Mois de } \\
\text { lactation }\end{array}$ & $\begin{array}{l}\text { Temps } \\
\text { total }\end{array}$ & $\begin{array}{l}\text { Temps du } \\
\text { débit maxi- } \\
\text { num }\end{array}$ & $\begin{array}{c}\text { Débit } \\
\text { maximum }\end{array}$ & $\% 3$ minutes & $\% 4$ minutes \\
\hline \multirow{4}{*}{ Valeurs brutes } & 2 & 6,3 & 1,6 & 2,3 & 76 & 86 \\
\hline & 5 & $5,{ }^{\prime}$ & 1,3 & 1,9 & 79 & 90 \\
\hline & 8 & $5,{ }^{\prime}$ & 1,6 & 1.7 & 76 & 89 \\
\hline & Total & 5,1 & $1,{ }^{\prime}+$ & 1,9 & 79 & 90 \\
\hline \multirow{4}{*}{$\begin{array}{c}\text { Valeurs corrigées } \\
\text { pour la quantité } \\
\text { de lait }\end{array}$} & 2 & 6,1 & 1,6 & 2,1 & 77 & 87 \\
\hline & 5 & 5,7 & $1,1^{\prime}$ & 2,1 & 78 & 88 \\
\hline & 8 & 6,2 & 1,7 & 2,0 & 73 & 85 \\
\hline & Total & 5,9 & 1,5 & 2,1 & $7 \mathrm{~s}$ & 89 \\
\hline
\end{tabular}

de mieux mesurer les aptitudes des animaux. Pour vérifier cette hypothèse nous avons calculé les coefficients de corrélation entre les contrôles aux $2^{\mathrm{e}}, 5^{\mathrm{e}}$ et $8^{\mathrm{e}}$ mois et la moyenne pour la lactation du même animal. Ces coefficients de corrélation ne dépendent pas de l'effet additif moyen du stade de lactation puisqu'ils ne font intervenir que les valeurs centrées des variables.

I)ans le cas où il n'existerait pas de différences entre les divers mois, on peut calculer le coefficient de corrélation moyen $(r)$ en fonction de la variance animal $\left(\sigma_{t}^{2}\right)$, de la variance résiduelle $\left(\sigma_{c}^{2}\right)$, et du nombre de contrôles $(n)$ pour la lactation totale. On a :

$$
\bar{r}=\frac{\sigma_{v}^{2}+\sigma_{e}^{2} / n}{\sqrt{\left(\sigma_{v}^{2}+\sigma_{e}^{2}\right)\left(\sigma_{v}^{2}+\sigma_{e}^{2} / n\right)}} .
$$

Cette relation peut être exprimée en fonction du coefficient de répétabilité $\mathrm{R}$ par la formule :

$$
\bar{r}=\sqrt{\mathrm{R}+(\mathrm{I}-\mathrm{R}) / n}
$$

Il est à remarquer que si le nombre de contrôles était très grand, on aurait $r=\sqrt{\mathrm{R}}$.

Dans l'échantillon des 27 génisses on avait $n=9$. Les coefficients de corrélation sont rassemblés dans le tableau if avec les valeurs correspondantes moyennes $\bar{\gamma}$. Pour calculer ces dernières nous avons pris comme coefficients de répétabilité ceux que nous avions obtenus à partir de la totalité des données. Le faible nombre des Ires lactations dont nous disposions ne nous a pas permis, en effet, d'obtenir des valeurs exactes.

Bien que notre effectif soit trop faible pour tirer des conclusions nettes, des tendances se dégagent cependant. Sans correction de la quantité de lait à la traite, seuls le débit maximum pendant les 5 premiers mois, et les pourcentages pendant 
toute la lactation donnent de bons résultats. Après correction, le temps total et les pourcentages donnent des valeurs analogues pendant toute la lactation, ainsi que le débit maximum pendant les 5 premiers mois. Comme SANdvik (I957 a) et Johansson (I96I), nous pouvons admettre qu'il est possible d'effectuer des contrôles de traite pendant au moins les 6 premiers mois de la lactation. Il serait souhaitable de vérifier si le débit maximum en fin de lactation a peu de signification ou si nos résultats ne sont dus qu'à des effets aléatoires.

TABLEAU I I

Coefficients de corrélation entre les contrôles situés à divers stades de lactation et la valeur moyenne pour la lactation

\begin{tabular}{|c|c|c|c|c|c|c|c|c|}
\hline & \multicolumn{4}{|c|}{ Variable non corrigée } & \multicolumn{4}{|c|}{ Variable corrigrée } \\
\hline & \multirow{2}{*}{ No mois } & \multicolumn{2}{|c|}{ Corrélation } & \multirow{2}{*}{$\mathrm{R}$} & \multirow{2}{*}{ No mois } & \multicolumn{2}{|c|}{ Corrélation } & \multirow{2}{*}{ R } \\
\hline & & Observée & 'Théoriquel & & & Observée & Théorique & \\
\hline Temps total & $\begin{array}{l}2 \\
5 \\
8\end{array}$ & $\begin{array}{l}0,39 \\
0,41 \\
0,27\end{array}$ & 0,65 & 0,58 & $\begin{array}{l}2 \\
5 \\
8\end{array}$ & $\begin{array}{l}0,60 \\
0,70 \\
0,63\end{array}$ & 0,66 & 0,61 \\
\hline $\begin{array}{l}\text { Temps débit } \\
\text { maximum }\end{array}$ & $\begin{array}{l}2 \\
5 \\
8\end{array}$ & $\begin{array}{l}0,35 \\
0,58 \\
0,62\end{array}$ & 0,56 & 0,18 & $\begin{array}{l}2 \\
5 \\
8\end{array}$ & $\begin{array}{l}0,37 \\
0,57 \\
0,65\end{array}$ & 0,56 & $0,{ }^{\prime}+8$ \\
\hline Débit maximum & $\begin{array}{l}2 \\
5 \\
8\end{array}$ & $\begin{array}{l}0,87 \\
0,77 \\
0,61\end{array}$ & 0,67 & 0,62 & $\begin{array}{l}2 \\
5 \\
8\end{array}$ & $\begin{array}{l}0,81 \\
0,75 \\
0,59\end{array}$ & 0,69 & $0,6 t^{\prime}$ \\
\hline $\begin{array}{l}\% 3 \text { premières } \\
\text { minutes }\end{array}$ & $\begin{array}{l}2 \\
5 \\
8\end{array}$ & $\begin{array}{l}0,82 \\
0,87 \\
0,77\end{array}$ & 0,76 & 0,72 & $\begin{array}{l}2 \\
5 \\
8\end{array}$ & $\begin{array}{l}0,8^{\prime} \\
0,8^{\prime} \\
0,81\end{array}$ & $0, \bar{i} 7$ & 0,74 \\
\hline $\begin{array}{l}\% 4 \text { premières } \\
\text { minutes }\end{array}$ & $\begin{array}{l}2 \\
5 \\
8\end{array}$ & $\begin{array}{l}0,8^{\prime} \\
0,8: 3 \\
0,66\end{array}$ & 0,73 & 0,69 & $\begin{array}{l}2 \\
5 \\
8\end{array}$ & $\begin{array}{l}0,85 \\
0,80 \\
0,71\end{array}$ & 0,73 & 0,69 \\
\hline
\end{tabular}

\section{7. - REIATIONS DES CRITÈRES DE TRAITE EITTRE EUX}

Les coefficients de corrélation linéaire entre les critères de traites sont représentés sur le graphique 6 . Le pourcentage de lait obtenu en 3 minutes est le plus lié aux autres critères et, en particulier au temps total. Par contre la liaison de ce dernier avec le débit maximum est particulièrement faible. L'analyse de la variance des valeurs moyennes des critères, les données étant groupées par classes de temps total, nous a permis de montrer que les régressions ne peuvent pas s'expliquer par des termes linéaires pour le débit maximum et les deux pourcentages. Cependant 


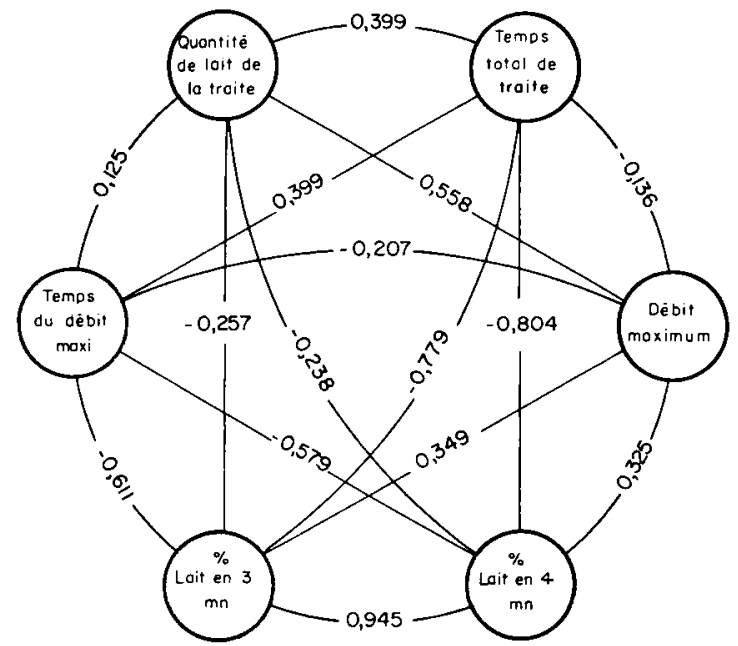

Graphique 6. - Corrélations totales entre les divers critères de traite

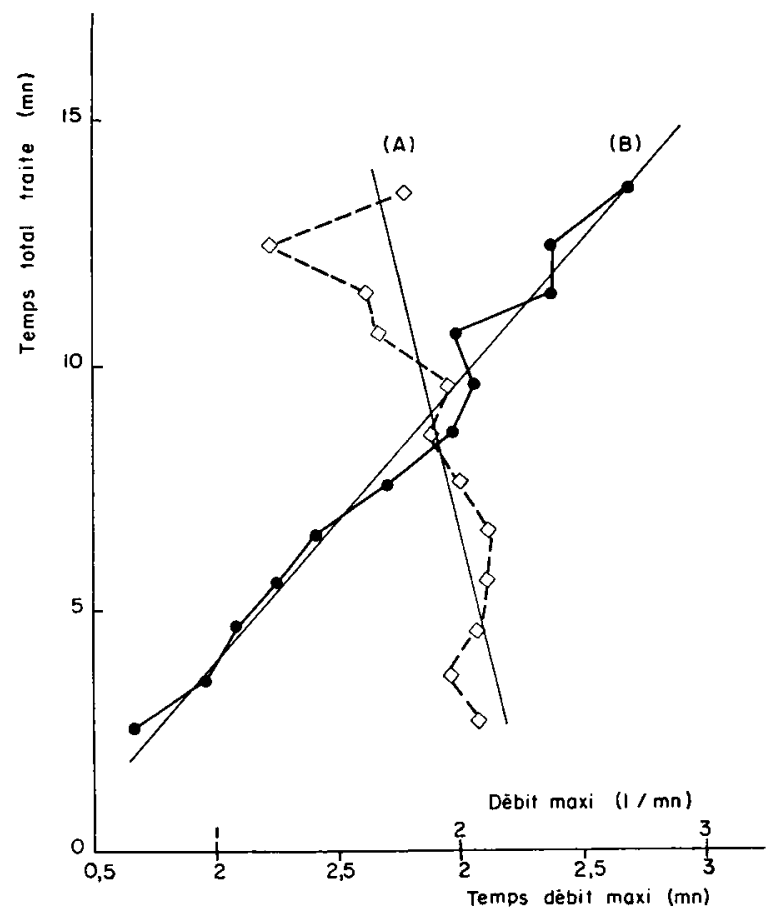

GRAPHIQUe 7. - Valeurs moyennes et droite de régression $\mathrm{A}-$ du débit maximum non corrigé,

$\mathrm{B}$ - du temps du débil maximum non corrigé en fonction du temps total de traite. 
l'augmentation du coefficient de corrélations que produirait un ajustement sur la courbe de régression estimée serait faible pour les pourcentages $(+0, \mathrm{OI})$ et encore peu élevée pour le débit maximum $(+0,06)$. La concordance entre la droite de régression et la courbe des valeurs moyennes est, d'ailleurs, satisfaisante (graph.7 et S).

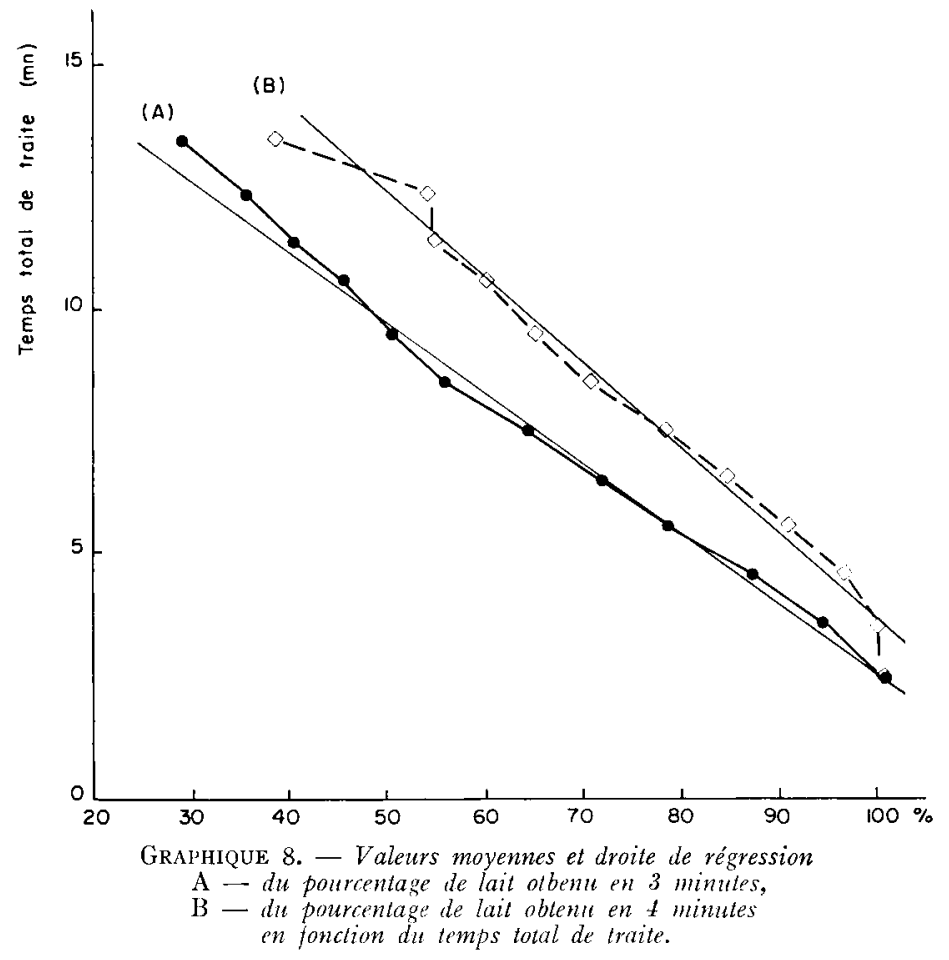

Les corrélations partielles, à production par traite constante, sont rassemblées dans le tableau I2.

TABLEAU I2

Corrélations partielles entre les caractères de traite, à production par traite constante

\begin{tabular}{|c|c|c|c|c|}
\hline & $\begin{array}{l}\text { l'emps du débit } \\
\text { maximum }\end{array}$ & Débit maximun & $\begin{array}{l}\% \text { lait en } \\
3 \text { minutes }\end{array}$ & $\begin{array}{l}\% \text { lait en } \\
1 \text { minutes }\end{array}$ \\
\hline $\begin{array}{l}\text { Temps total............. } \\
\text { 'lemps du débit maximum } \\
\text { Débit maxinum ......... } \\
\% \text { de lait en } 3 \text { minutes.... }\end{array}$ & 0,38 & $\begin{array}{r}-0,10 \\
-0,29\end{array}$ & $\begin{array}{r}-0,76 \\
-0,60 \\
0,52\end{array}$ & $\begin{array}{r}0,79 \\
-0,57 \\
0,18 \\
0,91\end{array}$ \\
\hline
\end{tabular}

Malgré l'augmentation très importante du coefficient de corrélation entre 1e débit maximum et le temps total, les pourcentages restent encore les critères les 
plus représentatifs de l'ensemble de la courbe de traite. Ici encore analyse statistique nous a montré que les relations entre le temps total de traite et le débit maximum ou les pourcentages ne sont pas linéaires. L'augmentation du coefficient de corrélation produite par l'ajustement des données sur la ligne de régression moyenne resterait cependant faible pour les pourcentages $(+0,02)$ et même pour le débit maximum $(+0,06)$. Il est intéressant de comparer dans ce cas la courbe

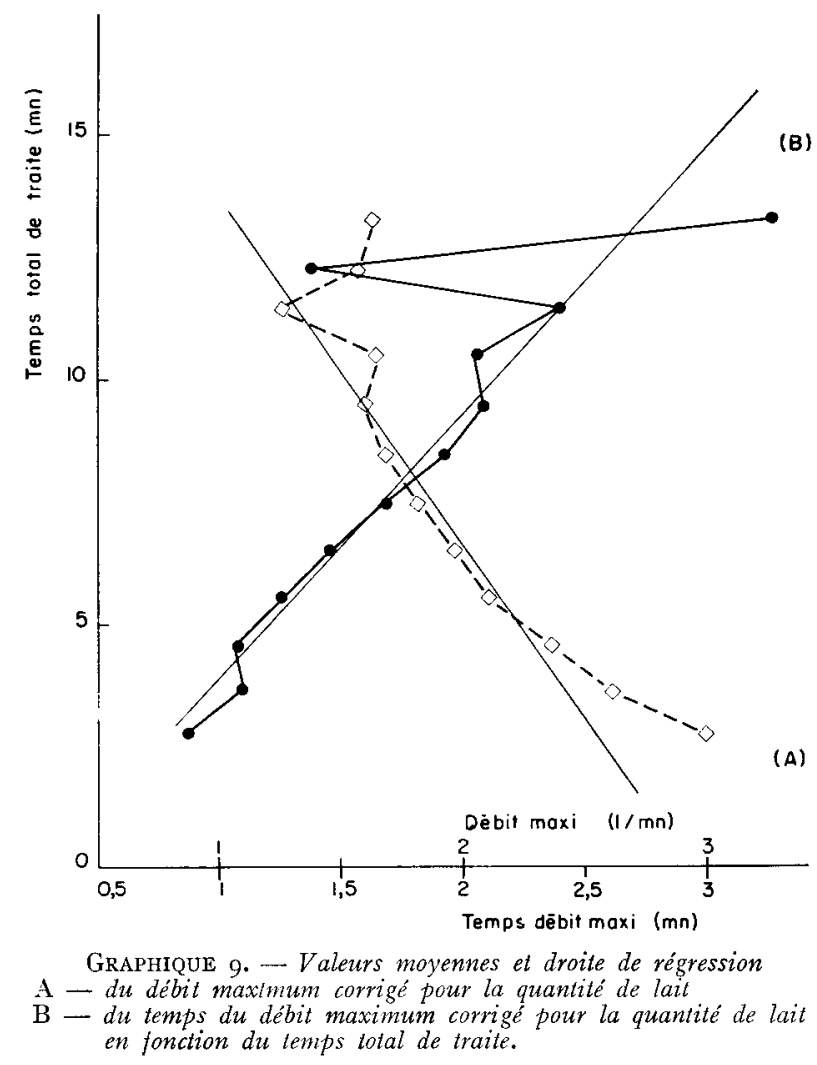

des valeurs moyennes à la droite de régression (graph. 9). Pour les pourcentages ces courbes sont pratiquement les mêmes que pour les données non corrigées par rapport à la quantité de lait.

Une sélection faite sur un pourcentage produirait un effet directement proportionnel sur le temps total de traite et ceci sur tout l'intervalle de variation possible. Au contraire l'augmentation du débit maximum au-delà de 3 litres par minute n'entrânerait qu' tune faible diminution du temps total. De ce point de vue il paraît donc préférable d'utiliser comme critère de choix des animaux, un pourcentage plutôt que le débit maximum. On doit cependant remarquer qu'il vaudrait mieux calculer le pourcentage de lait sur un temps plus réduit, 2 minutes par exemple (BECK et al., I95I) puisqu'il n'est pas possible de distinguer des animaux ayant une durée de traite inférieure à 3 ou 4 minutes suivant le cas. Il est à remarquer de plus que le coefficient de corrélation entre le débit maximum et le pourcentage 
de lait recueilli en 3 minutes est élevé $\left(r=0,5^{2}\right)$, de sorte qu'une sélection sur le dernier conduirait à éliminer les animaux ayant un débit maximum faible. Enfin, le coefficient de corrélation entre les pourcentages eux-mêmes $(r=0,94)$ est très grand comme on pouvait s'y attendre.

Si l'on introduit, dans 1'équation de prédiction du temps total de traite, la quantité totale de lait et le pourcentage de lait en 3 minutes, on explique $63 \mathrm{p}$. Ioo de la variance de ce critère. La mesure la plus intéressante à utiliser en troisième lieu serait le pourcentage de lait en 4 minutes, tandis que le débit et le temps du débit maximum n'entraîneraient qu'une amélioration négligeable du coefficient de corrélation multiple.

\section{8. - RELATIONS ENTRE LES CARACTÈRES DE TRAITE ETT DE PRODUCTION}

DODD et Foot (I953) ont trouvé une relation significative entre le débit maximum et la production par lactation (en 305 jours) et la persistance de cette dernière. Pour une augmentation du débit maximum de $\mathrm{I} \mathrm{kg} / \mathrm{mn}$, la production par lactation augmente de $400 \mathrm{~kg}$ et l'index de persistance de Io jours. Des résultats analogues ont été observés par plusieurs chercheurs (DoNAID, I960; JoHAxsson, Ig6I ; SANDVIK, I957 a). SANDVIK (1957 b) a cependant montré que si les corrélations sont calculées à production par contrôle constante, elles ne sont plus significatives. Nous avons calculé à partir de nos données les coefficients de corrélation de la production totale au cours de la lactation, et de la durée de la lactation avec les caractères de traite, la quantité de lait par contrôle étant, ou non, maintenue constante (tabl. I3).

\section{TABLEAU 13}

Caefficients de corrélation linéaire de la production et de la durée de la lactation avec les caractères de traite

\begin{tabular}{|c|c|c|c|c|c|}
\hline Corrélations totales & $\begin{array}{l}\text { Temps de } \\
\text { traite }\end{array}$ & $\begin{array}{l}\text { Tempss du } \\
\text { débit maxi- } \\
\text { mum }\end{array}$ & $\begin{array}{l}\text { Débit } \\
\text { maximum }\end{array}$ & $\begin{array}{l}\text { "lait en } \\
3 \text { minutes }\end{array}$ & $\begin{array}{l}\text { " lait en } \\
\text { iminutes }\end{array}$ \\
\hline $\begin{array}{r}\text { Production rle lait par } \\
\quad \text { lactation } \ldots \ldots \ldots \ldots \ldots\end{array}$ & $-0,09$ & $-0,233$ & 0,05 & 0,17 & 0,13 \\
\hline Durée de lactation....... & $-0,18$ & $-0,41$ & $-0,21$ & 0,51 & 0,43 \\
\hline $\begin{array}{c}\text { Corrélations partielles à } \\
\text { production totale par } \\
\text { contrôle constant }\end{array}$ & $\begin{array}{l}\text { Temps de } \\
\text { traite }\end{array}$ & $\begin{array}{l}\text { Temps du } \\
\text { débit maxi- } \\
\text { mum }\end{array}$ & $\begin{array}{c}\text { Débit } \\
\text { maximum }\end{array}$ & $\begin{array}{l}\% \text { lait en } \\
3 \text { minutes }\end{array}$ & $\begin{array}{l}\% \text { lait en } \\
\% \text { minutes }\end{array}$ \\
\hline $\begin{array}{r}\text { Production de lait par } \\
\text { lactation } \ldots \ldots \ldots \ldots \ldots \ldots\end{array}$ & $--0,12$ & $-0,23$ & 0,0 & 0,18 & 0,15 \\
\hline Durée de lactation....... & $-0,{ }_{1}{ }_{1} 0$ & $-0,10$ & 0,0 & 0,45 & 0,37 \\
\hline
\end{tabular}


Sur 1'échantillon analysé, les relations entre la production totale par lactation et les caractères de traite sont les mêmes que l'on tienne compte ou non de la quantité de lait par contrôle. Il n'est pas douteux que les vaches les plus faciles à traire sont également les plus productives. Néanmoins, la valeur des coefficients de régression est suffisamment faible pour que l'incidence pratique d'une telle liaison soit peu importante. Par contre, les corrélations entre certains caractères de traite et la durée de lactation sont très hautement significatives, même en tenant compte de la quantité de lait à la traite. Les tarissements précoces peuvent être expliqués par la présence dans la mamelle de quantités élevées de lait restant après la traite par suite d'un égouttage insuffisant ou d'une structure défectueuse de la mamelle ellemême. Cette dernière hypothèse paraît la plus probable, compte tenu des conditions d'exploitation de la Pierre-qui-Vire. Les animaux les plus longs à traire seraient aussi ceux qui ont naturellement la quantité de lait résiduel la plus élevée, ce qui diminuerait la durée de la lactation.

\section{CONCLUSION}

Toute sélection effectuée à grande échelle dans les conditions pratiques de contrôle suppose au préalable la mise au point d'une méthode de mesure simple, mais efficace. L'étude préliminaire faite sur les résultats de 4 années d'observations à la ferme de l'Abbaye de la Pierre-qui-Vire nous a permis de vérifier qu'il est possible de mettre en évidence des différences systématiques entre vaches. Ces différences dépendent de nombreuses causes de variation : quantité de lait à la traite, numéro de lactation, stade de lactation, technique de traite et valeur de la main-d'œuvre employées. Dans les conditions ot̀ nous avons opéré, les meilleures caractéristiques sont, dans l'ordre : le pourcentage de lait obtenu au cours des 3 premières minutes, le débit maximum et la durée totale de traite.

Les valeurs obtenues pour les répétabilités sont nettement inférieures à celles avancées, à ce sujet, par la plupart des auteurs. Nous pensons que la principale raison tient dans le caractère volontairement appliquée que nous avons donné à notre recherche; elle a eu lieu sur une ferme bien conduite, certes, mais dans les conditions mêmes de la pratique; les animaux ont été contrôlés I fois par mois, pendant 4 années consécutives, le personnel de contrôle n'étant pas spécialisé pour ce type de travail, et les conditions de traite n'ayant pas été parfaitement standardisées pendant cette période. Les résultats obtenus restent, malgré tout, très encourageants, puisqu'il suffirait de 4 contrôles par vie de l'animal, pour le caractériser de façon convenable (répétabilité de la moyenne du pourcentage en 3 minutes moyenne supérieure à 0,70 ).

Nos données n'étaient cependant pas suffisamment nombreuses pour nous permettre de fixer des règles de contrôle. En particulier, nous n'avons opéré que dans une seule ferme, alors que l'analyse faite tend à prouver que l'influence de la technique de traite est très importante ; on peut penser à juste titre qu'une très grande diversité existe, dans ce domaine, d'élevage à élevage. Une enquête portant sur un plus grand nombre d'animaux de 3 races différentes, répartis dans une ving- 
taines de fermes, est en cours d'exécution. Elle fournira des renseignements plus précis sur la meilleure méthodologie de contrôle et l'étendue de la sélection qu'il est souhaitable de pratiquer sur le caractère " aptitude à la traite ".

Rę̧u pour publication en janvier 1963.

\section{REMERCIEMENTS}

Nous tenons à remercier le R. P. Anschaire, Directeur de la ferme de la Pierre-qui-Yire, pour l'aide permanente qu'il nous a apportée au cours de la réalisation de nos travaux.

Nos remerciements vont également aux stagiaires qui ont collecté les données, ou qui ont collaboré à leur interprétation.

\section{SUMMARY}

\section{A PRELIMINARY STUDY OF THE MILKING CIIARACTERISTICS OF MILK COWS}

Tests were carried out from $195^{6}$ to 1959 on a herd of Brune des Alpes milk cows : 1207 samples from 77 different animals were analysed.

The criteria used were :

- total quantity of milk;

- total time of milking;

- maximum flow per minute ;

- date of maximum flow during the milking period;

- quantities of milk drawn during the first 3 and 4 minutes expressed as percentages of the total milking;

and the figures showed little inclination to repeat themselves (table 2 ). The chief factors likely to cause variations in these characteristics were also studied.

$\mathrm{I}^{\mathrm{o})}$ The total amount of milk drawn was strictly correlated with the other criteria (table 3 ), while a correction for the quantity of milk does not improve the repeatability (table 4).

$2^{\circ}$ ) The period during which the tests were carried out exercised an important influence upon the variations in this data, this being due to changes in milking conditions over the course of years. The characteristic least affected was the percentage of milk drawn during the first 3 minutes of milking (table 5).

$3^{\circ}$ ) The lactation number was one source of variation in the criteria studied (table 8), but its influence seemed to be exercised indirectly by its action on the total amount of milk drawn at the milking (table 9).

$\left.4^{\circ}\right)$ The stage of lactation had an influence which was not negligible on the milking characteristics; a test made during the first half of the period of lactation gave a better indication of the milking capacity of the animal (table is).

The relationships between the criteria themselves (graph. 6) and between the milking criteria and those of the lactation (table ${ }_{3}$ ) showed that it would be possible to envisage a selection based upon milking capacity which would not be contrary to a genetical improvement of milk production.

\section{RÉFÉRENCES BIBLIOGRAPHIQUES}

BeCK G. H., FRYER H. C., ROAK D. B., I95I. Use and interpretation of milk flow curves in measuring variations in the response to machine milking. J. Dairy Sci., 34, 58.67.

Compte rendu du VIII ${ }^{e}$ Congrès International de Zootechnie, Hambourg, Ig6r.

Compte rendu des Journées de l'Association Française de Zootechnie, Paris, ig6o. 
DodD F. H., I953. Normal variations in the rate of machine milking. J. Dairy Res., 20, 30I-3I8.

DoDD F. H., Foor A. S., 1953. The importance of machine milking rate in dairy cow management. $J$. Dairy Res., 20, 138-145.

Dodd F. H., Foot A. S., Henriques E., Neave F. K., I950. The effect of subjecting dairy cows, for a complete lactation, to a rigid control of the duration of milking. J. Dairy Res., 17, 108-116.

DoNald H. P., I960. Genetical aspects of maximum rate of flow during milking. J. Dairy Res., 27, 361-37 I.

Johansson L., I961. Genetic aspects of dairy cattle breeding. University of Illinois, Urbana, I 22-134.

Henderson C. R., 1953. Estimation of variance and covariance components. Biometrics, 2, $226-252$.

Polıtiek R. D., I96I. Beobachtungen über die Möglichkeit zur Ferstellung der Melkbarkeit und ihrer variation bei Kühen, auch im Hinblick auf die Heritabilität dieser Eigenschaft. Congrès F. E. Z. Hambourg I961, Gen. Rep., I48-ı66.

SaNDVIK O., I957. Different systems of judging the cow's milking characters in the selection of breeding animals. Meldinger tra Norges Landbrukshogskole, 36 (4), 1-26.

SANDVIK O, 1957. The relationship between milking rate and milk yield. J. Dairy Res., 24, 316-320.

WiLke G., 1960. Die Melkbarkeit ihre Abhängigkeit von der Euterform und ihr Einfluss and die Lakta* tions kurve. Z. Tierz. Züchtbiol., 74, 48-96. 